\title{
Lipid Characteristics of Pregnant Women With Subclinical Hypothyroidism and Their Correlation to the Pregnancy Outcomes
}

Jingjing Li

Zhengzhou University

Yajuan Xu ( $\nabla$ cnzzzsi@163.com)

Zhengzhou University

Zongzong Sun

Zhengzhou University

Yanjun Cai

Zhengzhou University

Biao Wang

Zhengzhou University

Miao Zhang

Zhengzhou University

Yanjie Ban

Zhengzhou University

Xiaofeng Hou

Zhengzhou University

Yingqi Hao

Zhengzhou University

Qian Ouyang

Zhengzhou University

Bo Wu

Zhengzhou University

Mengqi Wang

Zhengzhou University

Wentao Wang

Zhengzhou University

\section{Research Article}

Keywords: subclinical hypothyroiidism; lipidomics, pregnancy, pathogenesis 
Posted Date: April 21st, 2021

DOI: https://doi.org/10.21203/rs.3.rs-424258/v1

License: (c) (i) This work is licensed under a Creative Commons Attribution 4.0 International License. Read Full License 


\section{Abstract}

Subclinical hypothyroidism ( $\mathrm{SCH}$ ) in pregnancy has become an important complication of pregnancy. We used nontargeted lipidomics to compare differential metabolites between women with $\mathrm{SCH}$ and healthy women. The metabolic pathways of the differential metabolites were analyzed using the Kyoto Encyclopedia of Genes and Genomes (KEGG) database. We found: 1. Multivariate analysis revealed 143 lipid molecules with different levels between the $\mathrm{SCH}$ group and the control group. Based on fold change, 30 differential lipid metabolites were selected as potential biomarkers. 2. KEGG pathway enrichment analysis showed that the differential metabolites participate in such pathways as pathogenic Escherichia coli infection response, metabolic pathways, glycerophospholipid metabolism. 3. Correlation analysis showed that sphingomyelin (SM) and phosphatidylcholine (PC) were positively correlated with tumor necrosis factor-a (TNF-a), C-reactive protein (CRP), and interleukin-6 (IL-6), while phosphatidylglycerol $(\mathrm{PG})$, and phosphatidylinositol $(\mathrm{PI})$ were negatively correlated with them. And PG was positively correlated with birth weight. The lipid profile of pregnant women with $\mathrm{SCH}$ is significantly different from that of healthy pregnant women. Lipid molecules with differential metabolism, such as SM, PE, and PI, might be targets for further investigation of the pathogenesis of $\mathrm{SCH}$ in pregnancy and reduce the incidence of adverse pregnancy outcomes.

\section{Introduction}

Subclinical hypothyroidism $(\mathrm{SCH})$ in pregnancy refers to the elevation of serum thyroid-stimulating hormone (TSH) in pregnant women with normal levels of free thyroxine (FT4) and total T4. Patients with $\mathrm{SCH}$ usually do not have obvious clinical symptoms or physical signs, so the diagnosis of $\mathrm{SCH}$ depends mainly on laboratory examinations. The prevalence of $\mathrm{SCH}$ during pregnancy has been increasing year by year, and it has become an important complication of pregnancy. Its adverse effects on pregnancy outcomes are becoming clear, including spontaneous abortion ${ }^{[1]}$, preeclampsia ${ }^{[2]}$, placental abruption, premature rupture of membranes, low birth weight ${ }^{[3]}$, fetal distress, preterm birth ${ }^{[4]}$, and abnormal neuropsychological and intellectual development ${ }^{[5]}$. The pathogenesis of $\mathrm{SCH}$ remains unclear and might be associated with nitric oxide (NO) ${ }^{[6]}$, Nesfatin- ${ }^{[7]}$, gene silencing mediated by microRNAs ${ }^{[8]}$, inactivation and mutation of the thyroid-stimulating hormone receptor gene ${ }^{[9]}$, and the gut-thyroid axis ${ }^{[10]}$. In summary, $\mathrm{SCH}$ during pregnancy has high incidence, lacks definite symptoms and physical signs, and causes obvious adverse pregnancy outcomes. However, research on its pathogenesis is still lacking.

Although previous serum and urine metabolomic studies provided some clues about the pathogenesis of $\mathrm{SCH}$ at the metabolic level, the pathogenesis of $\mathrm{SCH}$ during pregnancy is still unclear, especially in terms of lipid metabolism. Zhang et al ${ }^{[11]}$ fed a high-fat diet (HFD) or chow diet to male Sprague-Dawley rats for 18 weeks. Their examination of thyroid function showed that HFD rats had hypothyroidism, and the liquid chromatography (LC)-mass spectrometry (MS) analysis showed that HFD rats had a disturbed thyroid lipid profile. Lee et al and Shao et al ${ }^{[12,13]}$ also pointed out that metabolic disorders induced by excessive nutrition might be a pathogenic factor of hypothyroidism, which revealed that the thyroid gland is another 
victim of lipotoxicity. The association between $\mathrm{SCH}$ and lipids has become more clear in recent years. What is unclear is how lipid metabolism disorders contribute to the pathogenesis of $\mathrm{SCH}$ during pregnancy. Therefore, the main purpose of this study was to use LC-MS to analyze the characteristics of the lipid profile of pregnant women with $\mathrm{SCH}$ in order to investigate its pathogenesis and reduce the incidence of adverse pregnancy outcomes.

\section{Results}

\section{General clinical data and pregnancy outcomes}

This study totally enrolled 30 patients in the $\mathrm{SCH}$ group and 30 patients at the same stage of pregnancy in the control group. The general clinical data of subjects in the two groups are shown in Table 1. The differences in age, BMI before pregnancy, BMI at enrollment, gravidity, parity, delivery mode, and Fasting blood glucose between the $\mathrm{SCH}$ group and the control group were not significant.

The pregnancy outcomes of subjects in the two groups are shown in Table 2. The differences in neonatal sex, Apgar scores at 1 and 5 minutes, between the $\mathrm{SCH}$ group and the control group were not significant, but birth weight (BW) was significant $(\mathrm{p}=0.036)$.

Table 1 Comparison of general clinical data between the $\mathrm{SCH}$ group and the control group

\begin{tabular}{|llll|}
\hline General Characteristics & SCH Group $(\mathrm{n}=30)$ & Control $(\mathrm{n}=30)$ & P-value \\
\hline Maternal age, year* & $30.57 \pm 4.21$ & $29.80 \pm 3.43$ & 0.443 \\
\hline BMI before pregnancy, $\mathrm{Kg} / \mathrm{m}^{2 *}$ & $21.62 \pm 2.92$ & $21.69 \pm 2.85$ & 0.921 \\
\hline $\mathrm{BMI}$ at enrollment, $\mathrm{Kg} / \mathrm{m}^{2 \star}$ & $25.95 \pm 5.72$ & $26.95 \pm 3.38$ & 0.410 \\
\hline Gravidity, $\mathrm{n}^{*}$ & $2.17 \pm 1.39$ & $1.97 \pm 1.35$ & 0.574 \\
\hline Parity, $\mathrm{n}^{*}$ & $0.40 \pm 0.50$ & $0.37 \pm 0.56$ & 0.808 \\
\hline delivery mode, $\mathrm{n}$ & & & 0.793 \\
\hline Normal delivery & 13 & 12 & \\
\hline Cesarean delivery & 17 & 18 & 0.792 \\
\hline FBG, mmol/L* & $4.59 \pm 0.59$ & $4.55 \pm 0.51$ & \\
\hline
\end{tabular}

*Data are expressed as means \pm standard deviation. $\mathrm{P}<0.05$ was considered statistically significant. 
Table 2 Comparison of pregnancy outcomes between the $\mathrm{SCH}$ group and the control group

\begin{tabular}{|llll|}
\hline General Characteristics & SCH Group $(\mathrm{n}=30)$ & Control $(\mathrm{n}=30)$ & P-value \\
\hline Neonatal sex & & & 0.795 \\
\hline Female, $\mathrm{n}$ & 13 & 14 & \\
\hline Male, $\mathrm{n}$ & 17 & 16 & \\
\hline Birth weight, ${ }^{\star}$ & $3213.53 \pm 289.10$ & $3374.80 \pm 294.35$ & 0.036 \\
\hline Apgar scores & & & \\
\hline 1 minute & $9.47 \pm 1.85$ & $9.70 \pm 0.65$ & 0.518 \\
\hline 5 minute & $9.60 \pm 1.83$ & $9.87 \pm 0.35$ & 0.436 \\
\hline
\end{tabular}

*Data are expressed as means \pm standard deviation. $\mathrm{P}<0.05$ was considered statistically significant.

\section{Difference analysis on the $\mathrm{SCH}$ group and the control group}

To validate the significant differences between the $\mathrm{SCH}$ group and the control group, the supervised partial least squares-discriminant analysis (PLS-DA) multivariate method was used for remodeling and analysis of the two groups of data (Fig. 1A). The results showed that most members of the two groups could be separated (R2Y-0.862 and Q2 $=0.286$ ). In model validation (Fig. 1B), R2 was $(0.0,0.74$ ) and Q2 was $(0.0,-0.55)$. The validation results showed that this model was stable and reliable. Differential lipid molecules were subjected to cluster analyses (Fig. 1C). The results showed that serum metabolites in these two groups had a certain aggregation trend.

\section{Screening of differential lipid molecules}

The differential lipid molecule screening conditions in this project were as follows: 1) a variable importance of projection score of the first two principal components of the PLS-DA model $\geq 1,2) F C \geq 1.2$ or $\leq 0.83$, and 3) t.test_p.value_BHcorrect $<0.05$. Compared with the healthy group, a total of 143 differential metabolites were identified in the $\mathrm{SCH}$ group, including 46 upregulated metabolites and 97 downregulated metabolites. The volcano plot used $\log _{2}(\mathrm{FC})$ as the $\mathrm{X}$-axis and $\log _{10}$ (t.test_p.value_BHcorrect) as the Y-axis (Fig. 2A).

Based on FC and biological significance, 30 compounds (the 15 most upregulated and 15 most downregulated) were studied as potential biomarkers (Fig. 2B). The upregulated compounds included sphingomyelin (SM), lysophosphatidylcholine (LPC), phosphatidylcholine (PC), and cholesteryl ester (ChE). The downregulated compounds included phosphatidylinositol (PI), lysophosphatidic acid (LPA), 
diglycerides (DG), lysophosphatidylethanol (LPEt), phosphatidylcholine (PC), phosphatidylethanolamine $(P E)$, phosphatidylglycerol (PG), and triglycerides (TG).

\section{Pathways analyses and correlation with clinical data and pregnancy outcomes of differential metabolites}

This study used the Kyoto Encyclopedia of Genes and Genomes (KEGG) database as the basis for linking differential metabolites to metabolic pathways, and enrichment analyses were performed on the pathways. The results indicated that differential metabolites in the $\mathrm{SCH}$ group and the control group participated in many pathways (Fig. 3A). Some of the main ones were the response to pathogenic Escherichia coli infection, regulation of lipolysis in adipocytes, metabolic pathways, glycerophospholipid metabolism, and fat digestion and absorption pathways.

Spearman correlation analyses were performed on the 29 differential metabolites that had the largest differences and clinical data (Fig. 3B). LPA and LPEt had a positive correlation with BMI at enrollment, while LPC had a negative correlation. SM, PC, and LPC showed a positive correlation with TNF-a, CRP, and IL-6, while PG, LPEt, LPA, PI, and different subclasses of PC showed a negative correlation with them. PG, LPA and TG had a positive correlation with BW, while LPC(20:0e) had a negative correlation. Moreover, there was no significant correlation between 29 differential metabolites and Apgar scores at 1 minute and 5 minute.

\section{Discussion}

Lipids are essential metabolites for living organisms and have many critical cellular functions. They can reflect the metabolic status of cells. Lipid metabolism in pregnant women changes significantly to meet the needs of growth and development of the fetus and ensure the health of the mother. If lipid metabolism is disordered, it will cause vascular endothelial damage, energy supply impairment ${ }^{[14]}$, and imbalance of immune functions and thyroid functions. This study performed a nontargeted plasma lipidomic analysis on pregnant women with $\mathrm{SCH}$ to investigate the pathogenesis of $\mathrm{SCH}$ during pregnancy.

The association between the thyroid gland and the lipidome is not well understood. Guidetti et al ${ }^{[15]}$ extracted total lipids from the mitochondria of rats with hypothyroidism and showed that the proportion of PC increased and the proportion of PE decreased. The LC-MS study of Liu et al ${ }^{[16]}$ showed that the levels of PC, SM, and PE in patients with hypothyroidism increased. Our PLS-DA plot showed that the lipidomes of pregnant women with $\mathrm{SCH}$ and healthy pregnant women were different. The volcano plot and the bar graph showed that the levels of PE, SM, and PC were higher in the SCH group than in the control group. The results are consistent with previous studies. Some studies ${ }^{[17,18]}$ have indicated that TSH and TG are positively correlated. Screening of differential metabolites between the two groups in our study showed that the levels of TG (16:0/16:1/18:2) and DG (16:0/16:0) decreased in the SCH group. The reasons for this discrepancy might be the small sample size and differences in experimental techniques. In addition, the TG (16:0/16:1/18:2) and DG (16:0/16:0) obtained in this study were subclasses of TG 
and DG and do not represent the serum levels of total TG and DG. Therefore, our results indicate that the lipidomes of the $\mathrm{SCH}$ group and the control group were significantly different, and these two groups could be distinguished by their lipid metabolism.

PE affects many cellular processes, including cellular stability and the functions of many membrane proteins, and is a critical regulatory factor of cell membrane fluidity. Our KEGG pathway analysis showed that differences in the pathogenic Escherichia coli infection response pathway were significant. In response to pathogenic Escherichia coli infection, during the contact process between bacterial flagellin and Toll-like receptor (TLR) 4 and TLR5 in gut epithelial cells, PE is an important stimulus of the Toll-like receptor signaling pathway to further induce inflammation. Our previous studies have shown that gut flora had significant differences between pregnant women with hypothyroidism and healthy pregnant women. The relative abundances of gut Proteobacteria, Gammaproteobacteria, and Provotella in women with hypothyroidism during pregnancy were all higher than those in the control group at the same stage. In recent years, changes in gut microbiota composition and function have been considered a hallmark of metabolic damage $\mathrm{e}^{[19]}$. The extremely diverse metabolites that are produced, such as lipopolysaccharides and endotoxins, could reduce the integrity of cell connections ${ }^{[20]}$. They enter the systemic circulation through the leaky gut mechanism to induce $T$ cell activation, resulting in a series of immune and inflammatory responses ${ }^{[21,22]}$. In addition, studies ${ }^{[23,24]}$ have indicated that inflammation and the TLR pathway are associated with thyroid functions.

SM levels are mainly altered by endoplasmic reticulum (ER) stress, and SM plays important physiological roles in cell growth, differentiation, senescence, and signal transduction ${ }^{[25]}$. ER stress ${ }^{[26]}$ might be a very important mechanism during the process of induction of SCH by a HFD, and the IRE1 $\mathrm{a} / \mathrm{XBP}-1 \mathrm{~s}$ pathway is involved. In this study, we observed that SM in the SCH group was higher than that in the control group and was positively correlated with IL-6. SM can be hydrolyzed by five different sphingomyelinases to exert various biological functions. Sphingomyelinases are influenced by TNF-a ${ }^{[27]}$ in vivo. Furthermore, the PI level in pregnant women with SCH during pregnancy was lower than that in healthy pregnant women. In thyroid cells, PI participates in intracellular TSH signal transduction through phosphatidylinositol $(3,4,5)$-trisphosphate (PIP-3) ${ }^{[28]}$. Lipid kinases in the phosphoinositide 3-kinase (PI3K) family can promote TH1 lymphocytes to produce higher levels of interferon-y and TNF-a secretion to stimulate release of chemokine ligand $(\mathrm{CXCL}) 10$ from thyroid cells to initiate and maintain the autoimmune process in the body ${ }^{[29-31]}$. In our correlation analysis, $\mathrm{PI}$ and TNF-a showed a negative correlation. SCH during pregnancy is an immune-metabolic disease. Zhu et al ${ }^{[32]}$ showed that IL-6 and TNF-a levels in SCH patients were significantly higher than those in healthy individuals. Therefore, metabolic disorders of SM and PI in pregnant women might cause $\mathrm{SCH}$ during pregnancy by affecting the autoimmune process.

In conclusion, we summarizes a possible pathogenic mechanism of SCH during pregnancy . $\otimes$ During the contact process between bacterial flagellin and TLR4 and TLR5 on gut epithelial cells, PE is an important stimulator of the TLR signaling pathway leading to inflammation and further influencing the development of the disease. $\otimes E R$ stress ${ }^{[26]}$ might be a very important mechanism during the process of induction of 
SCH by HFD. The IRE1a/XBP-1s pathway is involved, and SM plays an important role. $\otimes$ PI participates in intracellular TSH signal transduction through the PIP-3 signaling pathway ${ }^{[28]}$. Disruption of PIP-3 signal transduction further influences immune processes to affect thyroid functions.

Some studies have shown that hypothyroidism during pregnancy could increase the risk of premature delivery, low birth weight infants, fetal distress ${ }^{[3,4]}$. Labarre et al. ${ }^{[33]}$ found that maternal blood lipid level during pregnancy can affect the umbilical cord blood lipid group and its relationship with body weight. Our study found that PG, LPA and TG were positively correlated with birth weight, while LPC(20:0e) was negatively correlated, and there was a significant difference in birth weight between the $\mathrm{SCH}$ group and the control group. Therefore, we can speculate that PG and other differential lipid metabolism molecules can affected birth weight of the newborn by umbilical cord blood. The results of our study did not find any difference between the two groups of neonatal Apgar scores at 1 minute and 5 minute, and there was no correlation between the potential biomarkers and Apgar scores at 1 minute and 5 minute. Therefore, this study did not find a significant impact of gestational hypothyroidism on fetal distress.

The strengths of this study are as follows: $₫$ The influence of drugs on lipid disorders was controlled in all pregnant women in the $\mathrm{SCH}$ group before treatment. $\otimes$ This study also included clinical examination results at a time close to the sample collection. Therefore, the association between the serum lipid profiles and clinical characteristics in pregnant women was tested at the smallest possible interval. Of course, this study also had limitations: $\otimes$ The sample size was small. $\otimes$ This study had certain regional specificity due to factors such as dietary habits and geographical differences. $\otimes$ Because specimens were collected after the thyroid function disorders were discovered, no causal relationship between lipid metabolism disorder and thyroid function abnormality could be confirmed. $\nabla$ This study only included samples in late pregnancy before the subjects took medication. Therefore, the dynamic changes to serum lipid metabolism in the whole pregnancy and after drug treatment could not be studied. These considerations also provide direction for our future studies.

In short, we analyzed the serum lipidomes of two groups of pregnant women and found significant differences in the lipid profiles between pregnant women with $\mathrm{SCH}$ and healthy pregnant women. Lipid metabolism disorder in pregnant women might result in the development of $\mathrm{SCH}$ during pregnancy by affecting inflammation and immune responses through an ER stress mechanism, PIP-3 signaling pathway disruption, and gut flora metabolism. Lipid molecules with differential metabolism, such as SM, $\mathrm{PE}$, and $\mathrm{PI}$, could be studied for their roles in the pathogenesis of $\mathrm{SCH}$ during pregnancy and reduce the incidence of adverse pregnancy outcomes.

\section{Material And Methods}

Study subjects Pregnant women who received regular perinatal health care in the outpatient department of the Third Affiliated Hospital of Zhengzhou University and gave birth in the hospital between July 2019 and January 2020 were randomly selected. They included 30 pregnant women with SCH (SCH group) in 
late pregnancy and 30 healthy pregnant women (control group) in late pregnancy who met the inclusion criteria.

Inclusion criteria (1) The thyroid function levels of the SCH group met the diagnostic criteria in the 2017 guidelines of the American Thyroid Association for the diagnosis and management of thyroid disease during pregnancy and the postpartum ${ }^{[34]}$ and the reference-range criteria developed by the Department of Clinical Laboratory of the Third Affiliated Hospital of Zhengzhou University $(11.5<\mathrm{FT} 4<22.7 \mathrm{pmol} / \mathrm{L}$, $\mathrm{TSH}>4.0 \mathrm{mIU} / \mathrm{L}$, and thyroid peroxidase antibody $(\mathrm{TPOAb})<35 \mathrm{U} / \mathrm{mL}$ ). The control group included pregnant women who had normal thyroid function and did not have other obstetric complications. (2) All pregnant women were in the late pregnancy period.

Exclusion criteria The exclusion criteria were as follows: (1) Patients who had incomplete data. (2) Patients who had central hypothyroidism. (3) Patients who had other obstetric complications. (4) Patients who were taking antithyroid drugs or thyroid hormone replacement. (5) Patients who had severe systemic diseases or were recovering from thyroiditis. (6) Patients who took drugs that affected thyroid functions in the last 3 months, including metoclopramide, iodine supplements, domperidone, amiodarone, and lithium carbonate tablets. (7) Patients who had a medical history of liver diseases, malignant tumors, diabetes mellitus, and hereditary hyperlipidemia. (8) Patients who took lipid-lowering drugs in the last 3 months, such as fibrates, statins, or Xuezhikang capsules. (9) Patients who had had intestinal surgery. (10) Patients who were aged $<18$ years.

Note: Central hypothyroidism refers to hypothyroidism caused by reduced production and secretion of thyrotropin-releasing hormone or TSH resulting from a disorder of the hypothalamus or pituitary gland.

Statement: (i) All enrolled subjects volunteered and signed informed consent forms. This study was approved by the Ethics Committee of the Third Affiliated Hospital of Zhengzhou University. (ii) All methods were carried out in accordance with relevant guidelines and regulations.

Specimen collection All pregnant women fasted for 8-12 $\mathrm{h}$ before blood collection. A total of $5 \mathrm{~mL}$ blood from the median cubital vein was collected in ethylenediaminetetraacetic acid-coated tubes. After collection, the collection tube was gently inverted four times, wrapped in aluminum foil, and temporarily stored in a $4^{\circ} \mathrm{C}$ refrigerator. Blood samples were centrifuged within $2 \mathrm{~h}$ of sample collection in a lowtemperature centrifuge at $4^{\circ} \mathrm{C}$ and $1600 \times \mathrm{g}$ for $10 \mathrm{~min}$. The supernatant (plasma) after centrifugation was aliquoted into several centrifuge tubes and stored in a $-80^{\circ} \mathrm{C}$ freezer. Later, samples were sent to the BGI Group for lipidomic examination.

Data collection The basic data of the two groups of pregnant women, including body mass index (BMI) before pregnancy, BMI at enrollment, gravidity, parity, and delivery mode, neonatal sex, birth weight (BW), Apgar scores at 1 and 5 minutes, were collected. In addition, clinical data were collected during sample collection, including serum TSH, fasting blood glucose (FBG), CRP, IL-6, TNF-a. 
Major instruments and reagents Instruments used in this study included an ultrahigh-performance liquid chromatograph (UPLC) (Waters 2D UPLC, Waters, USA), a high-resolution mass spectrometer (Q Exactive, Thermo Fisher Scientific, USA), chromatographic column: ACQUITY UPLC CSH C18 $(1.7 \mu \mathrm{m} \varangle 2.1 * 100 \mathrm{~mm} \rrbracket$ Waters, USA), a low-temperature ultracentrifuge (Centrifuge 5430冈Eppendorf), a vortex (QL-901, Qilin Beier instrument, China), a water purifier (Milli-Q Integral『Millipore CorporationखUSA), and a refrigerated vacuum concentrator $\triangle$ Maxi Vacbeta囚GENE COMPANY\. Reagents used in this study included LC-MSgrade (Thermo Fisher Scientific $₫$ USA) methanol $₫ A 454-4 \rrbracket$, acetonitrile $₫ A 996-4 \rrbracket$, isopropanol (A461-4),

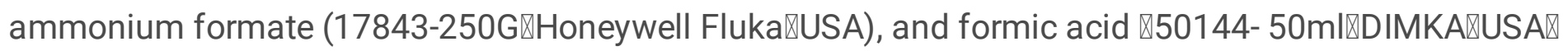
and water purified by a water purifier.

Extraction of lipid molecules After samples were slowly thawed at $4^{\circ} \mathrm{C}, 100 \mu \mathrm{L}$ of each sample was pipetted into a 96-well plate. Next, $300 \mu$ isopropanol (precooled to $-20^{\circ} \mathrm{C}$ ) and $10 \mu \mathrm{l} \mathrm{SPLASH} \mathrm{Lipidomix}$ internal standard were added and vortexed to homogeneity for $1 \mathrm{~min}$. Samples were kept still at $-20^{\circ} \mathrm{C}$ overnight and then centrifuged at $4^{\circ} \mathrm{C}$ and $4000 \mathrm{rpm}$ for $20 \mathrm{~min}$, The supernatant was collected and placed in a sample tube. The supernatant of each sample was collected at $10 \mu \mathrm{L}$ and mixed together to form the quality control (QC) sample.

UPLC-MS analysis UPLC materials and methods: The CSH C18 column $(1.7 \mu \mathrm{m} 2.1 * 100 \mathrm{~mm}$, Waters, USA) was used for chromatography. The mobile phases of the positive ion mode were a water solution (solution A) containing $10 \mathrm{mM}$ ammonium formate, $0.1 \%$ formic acid, and $60 \%$ acetonitrile and a solution (solution B) containing $10 \mathrm{mM}$ ammonium formate, $0.1 \%$ formic acid, $90 \%$ isopropanol, and $10 \%$ acetonitrile. The mobile phases of the negative ion mode were a water solution (solution $A$ ) containing 10 $\mathrm{mM}$ ammonium formate and $60 \%$ acetonitrile and a solution (solution $\mathrm{B}$ ) containing $10 \mathrm{mM}$ ammonium formate, $90 \%$ isopropanol, and 10\% acetonitrile. The following gradient was used for elution: 0-2 min, 40$43 \%$ solution $B$; $2 \sim 2.1 \min \varangle 43 \% \sim 50 \%$ solution $B \otimes 2.1 \sim 7 \min \varangle 50 \% \sim 54 \%$ solution $B \otimes 7 \sim 7.1 \min \varangle 54 \% \sim 70 \%$

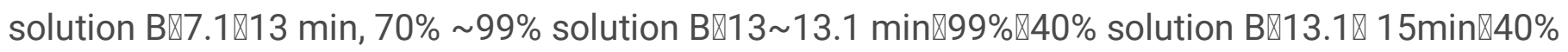
solution $\mathrm{B} \otimes$ The flow rate was $0.35 \mathrm{~mL} / \mathrm{min}$, the column temperature was $55^{\circ} \mathrm{C}$, and the sample load was $5 \mu \mathrm{L}$.

MS materials and methods: The Q Exactive mass spectrometer (Thermo Fisher Scientific, USA) was used for primary and secondary MS data acquisition. The range of the mass-to-charge ratio in MS scanning was 200-2000, the primary resolution was 70,000, the automatic gain control was $3 \mathrm{e} 6$, and the maximum injection time was $100 \mathrm{~ms}$. According to the precursor ion intensity, Top3 was selected for fragmentation. The secondary MS data were collected. The secondary resolution was 17,500, the automatic gain control was $1 \mathrm{e} 5$, the maximum injection time was $50 \mathrm{~ms}$, and the stepped normalized collisional energy was set as 15,30 , and $45 \mathrm{eV}$. The settings of electrospray ionization were a sheath gas flow rate of 40 , an aux gas flow rate of 10 , a spray voltage $(|\mathrm{kV}|)$ of 3.80 in the positive ion mode and 3.20 in the negative ion mode, a capillary temperature of $320^{\circ} \mathrm{C}$, and an aux gas heater temperature of $350^{\circ} \mathrm{C}$.

To provide more reliable experimental results during instrument detection, samples were randomly sorted to minimize systemic errors. One QC sample was inserted for every 10 samples. 
Statistical methods Raw data generated by the LC-MS/MS detection were input into LipidSearch v.4.1 (Thermo Fisher Scientific, USA) for MS data analysis. The data matrix contained the lipid molecule identification results and quantitative results. Identification and peak extraction of individual samples were first performed using LipidSearch v.4.1, and then peak alignment was performed on all samples. The raw data obtained from LipidSearch were input into metaX for data pretreatment and subsequent analyses. SPSS 25.0 software was used to process basic clinical data of pregnant women in two groups. Normally distributed quantitative data are described as mean \pm standard deviation $(\chi \pm s)$, and differences between groups were compared using the independent-samples t-test. Nonnormally distributed quantitative data are expressed as median $(M)$ and quartile $(Q)$, and differences between groups were compared using the Mann-Whitney U-test. Categorical variables are described as frequency, and the differences between groups were compared by the chi-squared test. Correlations were calculated by Spearman analysis.

\section{Declarations}

\section{Funding}

None.

\section{Author contributions}

Y.X. conceived and designed research; Y.X., Z.S., Y.C., B.W., M.Z., Y.B., X.H. Y.H., Q.O., B.W., M.W. and W.W. performed experiments; J.L.,Y.C. and Z.S. analyzed data; J.L., Y.X., Z.S., Y.C., B.W., M.Z., and Y.B.,. interpreted results of experiments; J.L. and B.W. prepared figures; J.L. drafted manuscript; J.L., Y.X., Z.S.,B.W., and M.Z. edited and revised manuscript; all authors approved final version of manuscript.

\section{Disclosures}

The author(s) declare no competing interests.

\section{Acknowledgments}

The authors are grateful to all patients and their parents who participated in this study.

\section{References}

1. Lee, S.Y. and E.N. Pearce, Testing, Monitoring, and Treatment of Thyroid Dysfunction in Pregnancy.J Clin Endocrinol Metab, 2020.

2. Mahadik, K., P. Choudhary, and P.K. Roy, Study of thyroid function in pregnancy, its feto-maternal outcome; a prospective observational study. BMC Pregnancy Childbirth, 2020. 20(1): p. 769.

3. Lee, S.Y., et al., Associations Between Maternal Thyroid Function in Pregnancy and Obstetric and Perinatal Outcomes. J Clin Endocrinol Metab, 2020. 105(5). 
4. Zhou, M., et al., Effects of thyroid diseases on pregnancy outcomes. Exp Ther Med, 2019. 18(3): p. 1807-1815.

5. Ge, G.M., et al., Maternal Thyroid Dysfunction During Pregnancy and the Risk of Adverse Outcomes in the Offspring: A Systematic Review and Meta-Analysis. J Clin Endocrinol Metab, 2020. 105(12).

6. Rong, S., et al., Nitric oxide is involved in the hypothyroidism with significant morphology changes in female Wistar rats induced by chronic exposure to high water iodine from potassium iodate. Chemosphere, 2018. 206: p. 320-329.

7. Xu, Y.Y., et al., High levels of Nesfatin-1 in relation to the dysfunction of the hypothalamic-pituitaryadrenal and hypothalamus-pituitary-thyroid axes in depressed patients with subclinical hypothyroidism. Neuropsychiatr Dis Treat, 2017. 13: p. 1647-1653.

8. Peixoto, T.C., et al., Early life nicotine exposure alters mRNA and microRNA expressions related to thyroid function and lipid metabolism in liver and BAT of adult wistar rats. Mol Cell Endocrinol, 2021. 523: p. 111141.

9. Moia, S., et al., The W520X mutation in the TSHR gene brings on subclinical hypothyroidism through an haploinsufficiency mechanism. J Endocrinol Invest, 2013. 36(9): p. 716-21.

10. Su, X., et al., Gut dysbiosis is associated with primary hypothyroidism with interaction on gut-thyroid axis. Clin Sci (Lond), 2020.

11. Zhang, X., et al., A High-Fat Diet Rich in Saturated and Mono-Unsaturated Fatty Acids Induces Disturbance of Thyroid Lipid Profile and Hypothyroxinemia in Male Rats. Mol Nutr Food Res, 2018. 62(6): p. e1700599.

12. Lee, M.H., et al., Thyroid dysfunction associated with follicular cell steatosis in obese male mice and humans. Endocrinology, 2015. 156(3): p. 1181-93.

13. Shao, S.S., et al., Dietary high-fat lard intake induces thyroid dysfunction and abnormal morphology in rats. Acta Pharmacol Sin, 2014. 35(11): p. 1411-20.

14. Luo, L. and M. Liu, Adipose tissue in control of metabolism. J Endocrinol, 2016. 231(3): p. R77-R99.

15. Giudetti, A.M., et al., Hypothyroidism down-regulates mitochondrial citrate carrier activity and expression in rat liver. Biochim Biophys Acta, 2006. 1761(4): p. 484-91.

16. Liu, J., et al., Serum Metabolomic Patterns In Patients with Autoimmune Thyroid Disease. Endocr Pract, 2020. 26(1): p. 82-96.

17. Hussain, A., et al., The Effects of Dyslipidemia in Subclinical Hypothyroidism. Cureus, 2019. 11(11): p. e6173.

18. Martinez Escude, A., et al., Relationship between hypothyroidism and non-alcoholic fatty liver disease in the Spanish population. Med Clin (Barc), 2020. 154(1): p. 1-6.

19. Sonnenburg, J.L. and F. Backhed, Diet-microbiota interactions as moderators of human metabolism. Nature, 2016. 535(7610): p. 56-64.

20. Farzi, A., E.E. Frohlich, and P. Holzer, Gut Microbiota and the Neuroendocrine System. Neurotherapeutics, 2018. 15(1): p. 5-22. 
21. Cani, P.D., et al., Involvement of gut microbiota in the development of low-grade inflammation and type 2 diabetes associated with obesity. Gut Microbes, 2012. 3(4): p. 279-88.

22. Power, S.E., et al., Intestinal microbiota, diet and health. Br J Nutr, 2014. 111(3): p. 387-402.

23. Turemen, E.E., et al., Endothelial dysfunction and low grade chronic inflammation in subclinical hypothyroidism due to autoimmune thyroiditis. Endocr J, 2011. 58(5): p. 349-54.

24. Yang, S.S., et al., The effects of subclinical hypothyroidism on serum lipid level and TLR4 expression of monocyte in peripheral blood of rats. Neuro Endocrinol Lett, 2014. 35(1): p. 80-6.

25. Birbes, $\mathrm{H}$., et al., Mitochondria and ceramide: intertwined roles in regulation of apoptosis. Adv Enzyme Regul, 2002. 42: p. 113-29.

26. Zhou, L., et al., Endoplasmic Reticulum Stress May Play a Pivotal Role in Lipid Metabolic Disorders in a Novel Mouse Model of Subclinical Hypothyroidism. Sci Rep, 2016. 6: p. 31381.

27. Al-Rashed, F., et al., Neutral sphingomyelinase 2 regulates inflammatory responses in monocytes/macrophages induced by TNF-alpha. Sci Rep, 2020. 10(1): p. 16802.

28. Fallahi, P., et al., Myo-inositol in autoimmune thyroiditis, and hypothyroidism. Rev Endocr Metab Disord, 2018. 19(4): p. 349-354.

29. Antonelli, A., et al., Chemokine (C-X-C motif) ligand (CXCL) 10 in autoimmune diseases. Autoimmun Rev, 2014. 13(3): p. 272-80.

30. Antonelli, A., et al., High values of Th1 (CXCL10) and Th2 (CCL2) chemokines in patients with psoriatic arthtritis. Clin Exp Rheumatol, 2009. 27(1): p. 22-7.

31. Antonelli, A., et al., Circulating chemokine (CXC motif) ligand (CXCL)9 is increased in aggressive chronic autoimmune thyroiditis, in association with CXCL10. Cytokine, 2011. 55(2): p. 288-93.

32. Zhu, C., et al., Reduction in Thyroid-Stimulating Hormone Correlated with Improved Inflammation Markers in Chinese Patients with Morbid Obesity Undergoing Laparoscopic Sleeve Gastrectomy. Obes Surg, 2019. 29(12): p. 3954-3965.

33. LaBarre, J.L., et al., Maternal lipid levels across pregnancy impact the umbilical cord blood lipidome and infant birth weight. Sci Rep, 2020. 10(1): p. 14209.

34. Alexander, E.K., et al., 2017 Guidelines of the American Thyroid Association for the Diagnosis and Management of Thyroid Disease During Pregnancy and the Postpartum. Thyroid, 2017. 27(3): p. 315-389.

\section{Figures}



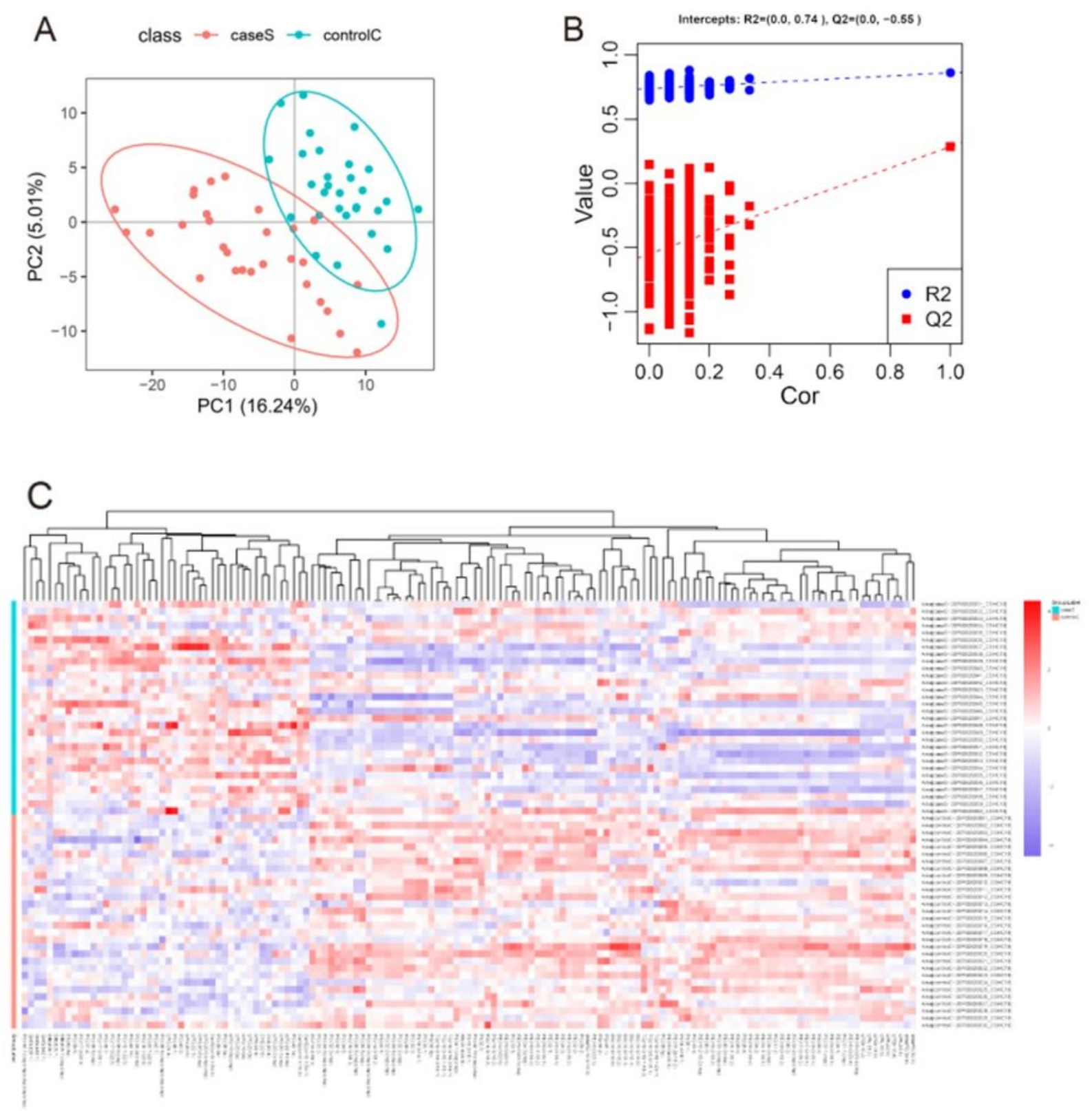

Figure 1

Difference analysis on the SCH group and the control group. A: the PLS-DA model score plot; B: response sequencing test plot of the PLS-DA analysis model. C: The cluster analysis diagram shows the aggregation trend in lipid profile between pregnancy group and the control group (Note: One point in the fifigure corresponded to one sample, green represented the control group, and red represented the $\mathrm{SCH}$ group. Each row in the graph represented a differential ion, and each column represented a sample. The 
different colors represented different intensities, of which the green meant intensity was low and the red meant intensity was high.)
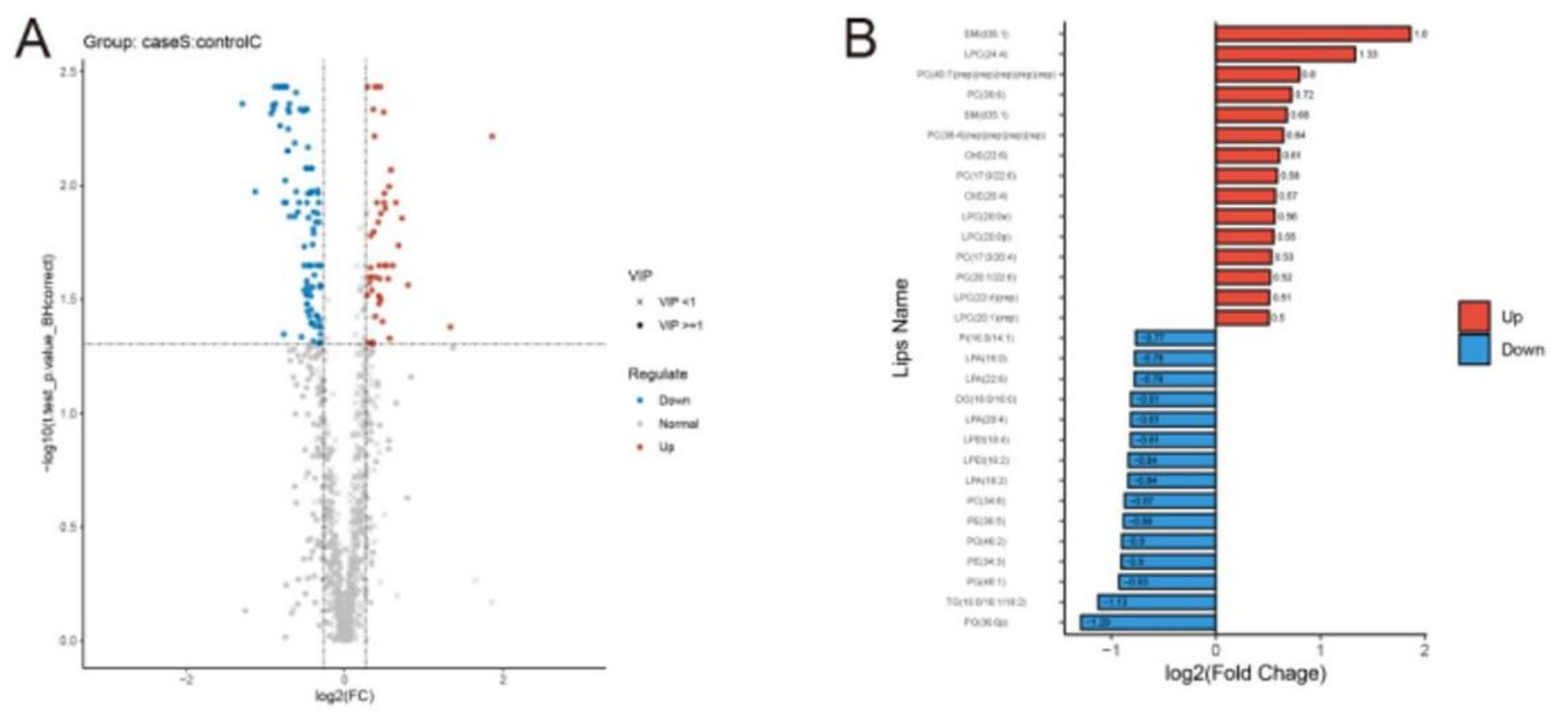

\section{Figure 2}

Screening of differential metabolites. A: Volcano plot shows the number of dysregulated lipid during $\mathrm{SCH}$ group compared to control group. B: Potential biomarkers (Note: Each row in the graph represented a differential ion, and each column represented a sample. Up: upregulation; Down: downregulation) 

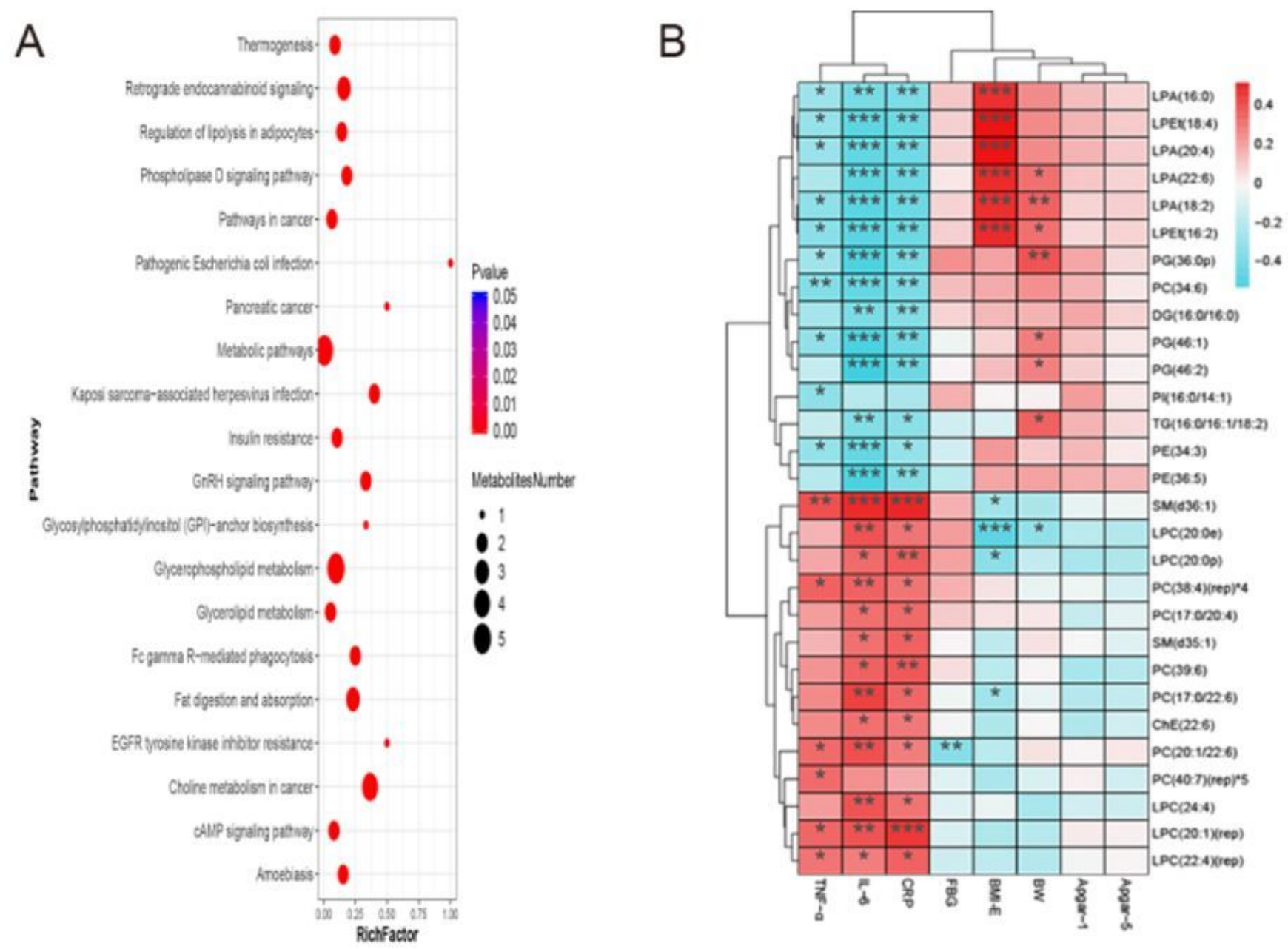

\section{Figure 3}

Pathways analyses and correlation with clinical data and pregnancy outcomes of differential metabolites. A:Scatter plot of KEGG enrichment. B: Correlation between potential biomarkers and clinical data.(Note: BMl: BMI at enrollment ; BW: birth weight; Apgar-1: Apgar scores at 1 minute; Apgar-5: Apgar scores at 5 minute; (rep): the identification results of this lipid were repeated. ${ }^{*}, p<0.1 ;{ }^{* \star}, p<0.01 ; * \star *$, $p<0.001$.) 